\title{
Food Digestibility by Microbes in Wild Ruminants: The Effect of Host Species and Dietary Substrate
}

\section{By Daniel E. Naya and William H. Karasov}

1 ood use by ruminant vertebrates strongly depends on the action of symbiotic microbes, because microbial fermentative digestion helps unlock energy from the single most abundant organic compound on earth, that is, cellulose in plant cell walls. In ruminant digestive chambers, a variety of cellulolytic bacteria, protists, and fungi transform cellulose, and also other cell wall materials, into soluble compounds that are then fermented., ${ }^{1,2}$ Likewise, microbes have played a keystone role in shaping the evolution of biodiversity of vertebrates: gut microbes made possible herbivory and a huge diversification of animal species into various feeding niches. ${ }^{3}$

It is noteworthy that with the advent of new molecular techniques we are learning more and more about this important symbiosis. ${ }^{4}$ Recent research on rumen microbial diversity, based on 16S rRNA gene sequences, suggests that there are at least 10 times the number of bacterial operational taxonomic units (OTUs) than previously suggested from culture-based methods. ${ }^{5}$ In addition, it seems that every time the gut microbial community of a new vertebrate herbivore is analyzed, the fermentative chamber-be it foregut, cecum, or colon-contains a high proportion of apparently previously undescribed OTUs. For example, a recent survey across 60 mammal species reported that on average $62 \%$ of OTUs within guts of mammalian hosts were not observed in any other species. ${ }^{6}$ Nevertheless, to date it is not clear how this huge diversity in gut microbial communities is linked with physiological variables, such as food digestion efficiency.

Ruminant nutritional ecologists often rely on in vitro fermentation by gut inocula of plant material to characterize the digestive efficiency of a donor species (i.e., consumer host) on the particular plant or plant mix. ${ }^{7}$ The in vitro dry matter digestibility (ivDMD) thus characterizes a performance feature of the gut microbial community that has been drawn from a particular host animal that has been maintained on a particular maintenance diet, which may or may not be the same as the test plant material, which we will refer to as dietary substrate. In this paper, we conducted a metaanalysis of previously published data for wild ruminant mammals to evaluate the effect of inocula donor species and the dietary substrate tested on ivDMD. Meta-analysis is considered an excellent method of comparing and summarizing the results of multiple studies for several reasons. ${ }^{8}$ One of these reasons is that it allows a highly improved control of Type II statistical errors (i.e., failure to reject a false null hypothesis), by combining the results of many studies. This feature is particularly important for the case under study, given that digestibility estimation in vertebrate herbivores sometimes employs relatively few (i.e., between one and three) animals.

\section{Methods \\ Database and Hypotheses}

We found a total of 16 studies, published between 1975 and 2005 , that reported data on ivDMD for wild ruminant species useful for our analysis (Table S1 in the online supplemental materials at http://dx.doi.org/10.2111/RANGELANDS-D-1000072.s1. All the data were obtained by the two-stage rumen liquor incubation method. ${ }^{9}$ We choose this methodology because it has been widely applied to wild ruminant species to provide an estimation of in vivo dry matter digestibility. ${ }^{7,10}$ The total numbers of comparisons on ivDMD retrieved from these papers was 89 (Table S1).

Our null hypotheses were that there are no differences in ivDMD between consumer species or between different dietary substrates within each consumer species. As one alternative to these, we propose the following hypothesis: 1) Microbial communities in guts of ruminants are from a functional perspective all rather similar across host species, but ivDMD differs among dietary substrates because of differences in chemical composition that influence rates of microbial degradation (e.g., cell wall content or composition, natural 
toxins, etc.). Under this alternative hypothesis we predict strong differences in ivDMD between dietary substrates, but no differences between ruminant species. Another alternative hypothesis is this: 2) Microbial communities in guts of ruminants are functionally different across host species, but the functioning of their microbial community is fairly independent of the dietary substrate. Under this hypothesis we predict strong differences in ivDMD between ruminant species, but not between dietary substrates.

Three points should be noted regarding our hypotheses. First, given that ivDMD could be affected by several factorse.g., animals' condition (e.g., gender, age, physiological state), methodological aspects (e.g., animal feeding regimen, time elapsed from the inoculum collection, time of the day of sample collection), and differences in the composition of the same (nominal) dietary substrates-we only compare results on species or dietary substrates from within the same study. Note that this constraint precludes us from using modern phylogenetic techniques to analyze differences between host species. Second, taking into account that very few studies simultaneously report data for different species and different dietary substrates, the interaction between both factors cannot be evaluated. Third, given that most of the studies on wildlife species meta-analyzed here used a domestic ruminant species as the control treatment or compare data for more than one wild and (or) domestic species, the effect of donor species on ivDMD was also analyzed separately for the following subcategories: domestic vs. domestic, wild vs. domestic, and wild vs. wild species.

\section{Data Analysis}

To test the above mentioned hypotheses we conducted a meta-analysis. We first calculated the effect size-as the difference between control and experimental mean values of ivDMD expressed in units of pooled standard deviation and corrected for small sample bias-and its associated variance for each comparison. ${ }^{11,12}$ In those cases in which sample size was equal to one or where errors were not reported we grouped data (e.g., dietary items, different seasons) to obtain an error estimation. Then, we estimated the cumulative effect size for each factor (i.e., donor species or dietary substrate), its associated variance, and the confidence interval for an alpha value of 0.05 , using a bootstrapping procedure with 1,000 iterations. ${ }^{11,12} \mathrm{~A}$ factor can be considered statistically significant if the $95 \%$ confidence interval around its cumulative effect size excludes zero. A conventional interpretation of the magnitude of an effect size is this: 0.2 represents a "small" effect, 0.5 represents a "medium" effect, 0.8 represents a "large" effect, and values greater than 1.0 are considered to represent a "very large" effect. ${ }^{13}$ Finally, to evaluate differences in cumulative effect size $(E)$ among factors, we used mixed-effects meta-analytical models plus heterogeneity tests. ${ }^{11,12}$ In these analyses, total heterogeneity $\left(Q_{T}\right)$ was decomposed into the heterogeneity

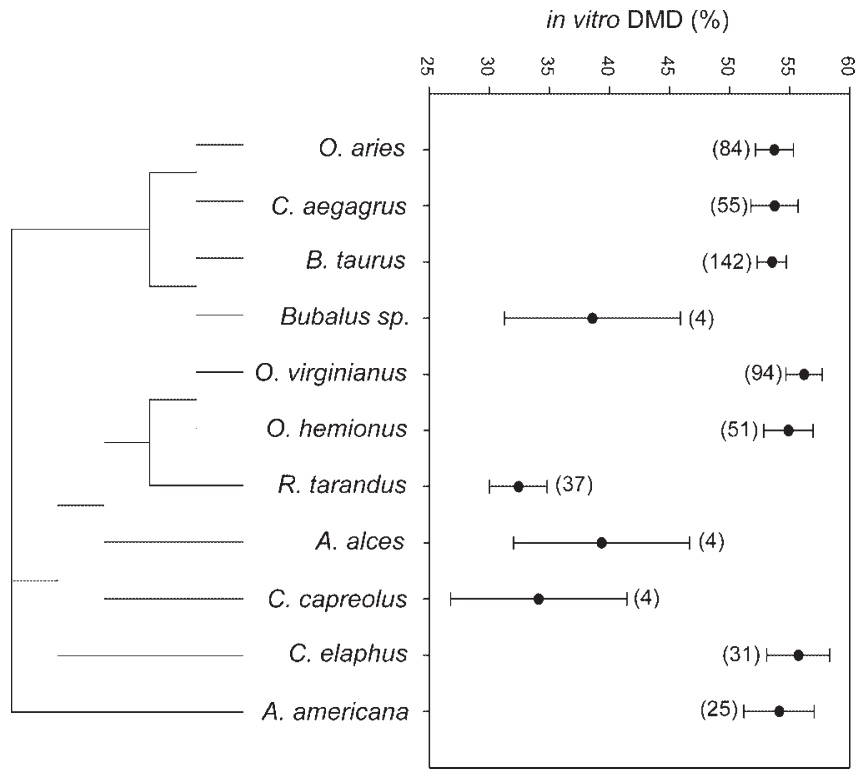

Figure 1. In vitro dry matter digestibility (DMD) for three domestic and eight wild ruminant species. Sample size (i.e., number of estimations) is given among brackets and bars represent $1 \mathrm{SE}$. The phylogenetic relationships among species (based on tree of life Web project, http:// tolweb.org/) are represented by a phylogenetic tree on the left side of the graph. Complete species names are given in Table 1 footnote.

explained by the model $\left(Q_{M}\right)$ and error heterogeneity $\left(Q_{E}\right)$, in a similar fashion as in a 1-way analysis of variance. Both $Q_{M}$ and $Q_{E}$ can be tested against a $\chi^{2}$ distribution with $m-$ 1 degrees of freedom for $Q_{M}$ and $n-m$ degree of freedom for $Q_{E}$ (where $n$ is the number of comparisons and $m$ the number of factors). A significant $Q_{M}$ means that at least one factor is significantly different in its cumulative effect size from the other factors. Analyses were conducted using METAWIN ${ }^{\circledR}$ version 2.0. ${ }^{11}$

\section{Results}

Although ivDMD ranged from $34.1 \%$ to $56.2 \%$, seven of the 11 species analyzed in this study showed values that were between $53.0 \%$ and $56.2 \%$ (Fig. 1). This relative similarity was particularly noticeable for the domestic species, for which ivDMD was $53.7 \%$ in goats and sheep and $53.5 \%$ in cows (Fig. 1). The remaining four species-buffalo (Bubalus sp.), reindeer (Rangifer tarandus), moose (Alces alces), and roe deer (Capreolus capreolus) - showed lower ivDMD values, ranging between $32.0 \%$ and $39.0 \%$ (Fig. 1).

Regarding the effects of donor species and dietary substrates, a significant difference between cumulative effect size of these two factors was found $\left(Q_{87}=51.76, P \leq 0.001\right)$. Specifically, it was observed that donor species did not affect ivDMD values, whereas dietary substrate had a large effect on this variable (Table 1). In the same vein, no differences were observed between donor species in ivDMD when comparing among domestic species, among wild species, or between domestic species and wild species $\left(Q_{60}=1.00\right.$, $P=0.61$; Table 1). 


\begin{tabular}{|c|c|c|c|}
\hline & $E$ & $95 \% \mathrm{Cl}$ & $n$ \\
\hline Donor species & 0.049 & $-0.078-0.182$ & 63 \\
\hline Domestic vs. domestic & -0.100 & $-0.222-0.047$ & 11 \\
\hline Domestic vs. wild & 0.088 & $-0.055-0.239$ & 39 \\
\hline Wild vs. wild & 0.001 & $-0.335-0.411$ & 13 \\
\hline Dietary substrate & 1.527 & $1.150-1.978$ & 26 \\
\hline
\end{tabular}

Note: Species refers to the kind of host consumer species, where domestic ruminant species include cow (Bos taurus), goat (Capra aegagrus), and sheep (Ovis aries) and wild ruminant species include moose (Alces alces), pronghorn antelope (Antilocapra americana), buffalo (Bubalus sp.), roe deer (Capreolus capreolus), red deer (Cervus elaphus), mule deer (Odocoileus hemionus), white-tailed deer (Odocoileus virginianus), and reindeer (Rangifer tarandus).

\section{Discussion}

Ten years ago, Kitessa and coworkers qualitatively reviewed the evidence on ivDMD for domestic ruminant animals, reaching the conclusion that the effect of donor species "is most likely to be small." ${ }^{\prime \prime}$ In line with this result, we found fairly similar values of ivDMD between wild donor species in paired comparisons.

On the other hand, we found a strong effect of the dietary substrate (within host species) on ivDMD. This result is not surprising given the variability of the substrates evaluated, which ranges from lichens to leaves of shrubs to commercial rations. However, a cautionary remark regarding this result should be made. Because most studies did not acclimate animals with the same diets that then were tested (i.e., maintenance diets were not the same as the test substrate), observed differences can be due, to some extent, to this fact. The functioning of the microbial community of the foregut is capable of changing according to the diet consumed (e.g., changed level of expression of some enzymes within microbe individuals or changes in the relative abundance of different microbes species in the community), and food digestibility can be affected by factors that need some time for adjustment. ${ }^{2,14}$ Nevertheless, studies showing a good correlation between ivDMD for different substrates and in vivo digestibility for the same substrates without regard to the acclimation diet ${ }^{15,16}$ suggest that differences between digestibilities of dietary substrates are real and not just an artifact due to the lack of acclimation.

Only four of the 11 host species evaluated here showed ivDMD mean values that were out of the narrow range between $53 \%$ and $58 \%$. It seems likely to us that this relates to the particular dietary substrates that were used. In the case of buffalo (Bubalus sp.) it is clear that the lower ivDMD values were related to the use of a low-quality plant material (Acacia saligna leaves), given that cows and sheep that were simultaneously evaluated also showed low digestibility values. ${ }^{17}$ For the other three species-reindeer, moose, and roe deer-lower ivDMD values are also likely related to the use of poor-quality substrates, because in most of the studies dietary items evaluated are very recalcitrant to breakdown (e.g., leaves of trees and shrubs, lichens, and mosses). When less recalcitrant materials were used, observed ivDMD values were similar to those measured in other species (e.g., $67.4 \%$ for reindeer on cattle ration, and $58.4 \%$ for moose on alfalfa hay).

As we mentioned in the introduction, there is a large variability in the in gut microbial community composition among vertebrate species ${ }^{18}$ including ruminant mammals. ${ }^{6}$ However, results obtained here indicate that, beyond these microbial community compositional differences, there appears to be a great degree of functional redundancy that is reflected in the microbial community's efficiency in breaking down substrate. This result is in line with recent metagenomic data, which suggest that beyond the great differences in microbiomes' phylogenetic composition between host species, and even between individuals of the same host species, there appear to be remarkable similarities in the pool of genes present in the microbiome of each species. ${ }^{19}$ Karasov et al. ${ }^{19}$ hypothesized that a shared core metagenome should exist for those host species that consume similar diets (e.g., herbivorous animals). Notwithstanding this very general statement, there are some notable examples where the possession of a particular microbial symbiont with an apparently particular physiological capability is associated with a dramatic functional difference at the level of the host. A classic example of this is the bacterium Synergistes jonesii, which is capable of degrading mimosine metabolites and imparts mimosine resistance in the host ruminant, allowing it to eat Leucaena spp. ${ }^{3}$

\section{Implications}

Our results have a practical significance besides offering insight into the structure-function relationships of microbial communities in guts of wild ruminants. Wildlife biologists sometimes use ivDMD procedures to estimate the nutritional value of wild foods. ${ }^{10}$ Current guidance suggests that the source of rumen fluid is an important factor in applying these procedures, based on studies that provide interesting and suggestive data but which usually suffer from some statistical shortcomings, such as reduced sample size. Our meta-analysis, which offers an alternative statistical procedure for evaluating this issue, indicates that so far there is not strong evidence that source of rumen fluid is an important factor in estimation of ivDMD of browse of wild ruminants and serves as a stimulus for more research on this topic with statistically robust experimental designs.

\section{References}

1. Dehority, B. A. 1993. Microbial ecology of cell wall fermentation. In: H. G. Jung, D. R. Buxton, R. D. Hatfield, and 
J. Ralph [EDs.]. Forage cell wall structure and digestibility. Madison, WI, USA: American Society of Agronomy, Crop Science Society of America, Soil Science Society of America. p. 425-449.

2. Karasov, W. H., and C. Martínez del Río. 2007. Physiological ecology. Princeton, NJ, USA: Princeton University Press. $741 \mathrm{p}$.

3. Karasov, W. H., and H. V. Carey. 2009. Metabolic teamwork between gut microbes and hosts. Microbe 4:323-328.

4. Peterson, D. A., D. N. Frank, N. R. Pace, and J. I. Gordon. 2008. Metagenomic approaches for defining the pathogenesis of inflammatory bowel diseases. Cell Host and Microbe 3:417-427.

5. Edwards, J. E., S. A. Huws, E. J. Kim, M. R. F. Lee, A. H. Kingston-Smith, and N. D. Scollan. 2008. Advances in microbial ecosystem concepts and their consequences for ruminant agriculture. Animal 2:653-660.

6. Ley, R. E., M. Hamady, C. Lozupone, P. J. Turnbaugh, R. R. Ramey, J. S. Bercher, M. L. Schlegel, T. A. Tucker, M. D. Schrenzel, R. Knight, and J. I. Gordon. 2008. Evolution of mammals and their gut microbes. Science 320: 1647-1651.

7. Kitessa, S., P. C. Flinn, and G. G. Irish. 1999. Comparison of methods used to predict the in vivo digestibility of feeds in ruminants. Australian Journal of Agricultural Research 50: $825-841$.

8. Arnevist, G., and D. Wooster. 1995. Meta-analysis: synthesizing research findings in ecology and evolution. Trends in Ecology and Evolution 10:236-460.

9. Tilley, J. M., and R. A. Terry. 1963. A two-stage technique for the in vitro digestion of forage crops. Journal of the British Grassland Society 18:104-111.

10. Haufler, J. B., and F. A. Servello. 1994. Techniques for wildlife nutritional analyses. In: T. A. Bookhout [ED.]. Research and management techniques for widlife and habitats. 5th ed. Bethesda, MD, USA: The Wildlife Society and Lawrence, KS, USA: Allen Press. p. 307-323.

11. Rosenberg, M. S., D. C. Adams, and J. Gurevitch [computer program]. 2000. MetaWin. statistical software for meta-analysis. Version 2.1. Sunderland, MA, USA: Sinauer Associates.
12. Gurevitch, J., And L. V. Hedges. 2001. Meta-analyses: combining the results of independent experiments. In: S. M. Scheiner and J. Gurevitch [EDs.]. Analysis of ecological experiments. New York, NY, USA: Oxford University Press. p. 347-369.

13. Cohen, J. 1969. Statistical power analysis for the behavioral sciences. New York, NY, USA: Academic Press. 415 p.

14. Huston, J. E., B. S. Rector, W. C. Ellis, and M. L. Allen. 1986. Dynamics of digestion in cattle, sheep, goats and deer. Journal of Animal Science 62:208-215.

15. Robbins, C. T., P. J. Van Soest, W. W. Mautz, and A. N. Moen. 1975. Feed analyses and digestion with reference to white-tailed deer. Journal of Wildlife Management 39:67-78.

16. Palmer, W. L., R. L. Cowan, and A. P. Ammann. 1976. Effect of inoculum source on in vitro digestion of deer foods. Journal of Wildlife Management 40:301-307.

17. Salem, A. F. Z. M. 2005. Impact of season of harvest on in vitro gas production and dry mass degradability of Acacia saligna leaves with inoculum from three ruminant species. Animal Feed Science and Technology 123/124:67-79.

18. Rawls, J. F., M. A. Mahowald, R. E. Ley, and J. I. Gordon. 2006. Reciprocal gut microbiota transplants from zebrafish and mice to germ-free recipients reveal host habitat selection. Cell 127:423-433.

19. Karasov, W. H., C. Martínez del Rio, and E. CaviedesVIDAL. 2011. Ecological physiology of diet and digestive systems. Annual Review of Physiology (in press).

Authors are Assistant Professor, Departamento de Ecología y Evolución, Facultad de Ciencias and Centro Universitario de la Regional Este, Universidad de la República, Montevideo 11400, Uruguay, and Research Associate, Center for Advanced Studies in Ecology and Biodiversity, Santiago de Chile 6513677, Chile, dnaya@fcien.edu.uy (Naya); and Professor, Dept of Forest and Wildlife Ecology, University of Wisconsin, Madison, WI 53706, USA (Karasov). Research was funded by Comision Sectorial de Investigación Cientifica (Uruguay) and FONDAP 1501-0001 Program 1 (Conicyt, Chile) to DEN, and US National Science Foundation (IOB-0615678) to WHK. 